\title{
Efficient Multilateration Tracking with Concurrent Offset Estimation using Stochastic Filtering Techniques
}

\author{
Patrick Dunau, Ferdinand Packi, Frederik Beutler, and Uwe D. Hanebeck \\ Intelligent Sensor-Actuator-Systems Laboratory (ISAS), \\ Institute for Anthropomatics, \\ Karlsruhe Institute of Technology (KIT), Germany. \\ \{patrick.dunau, ferdinand.packi, frederik.beutler\}@kit.edu, and uwe.hanebeck@ieee.org
}

\begin{abstract}
Multilateration systems operate by determining distances between a signal transmitter and a number of receivers. In aerial surveillance, radio signals are emitted as Secondary Surveillance Radar (SSR) by the aircraft, representing the signal transmitter. A number of base stations (sensors) receive the signals at different times. Most common approaches use time difference of arrival (TDOA) measurements, calculated by subtracting receiving times of one receiver from another. As TDOAs require intersecting hyperboloids, which is considered a hard task, this paper follows a different approach, using raw receiving times. Thus, estimating the signal's emission time is required, captured as a common offset within an augmented version of the system state. This way, the multilateration problem is reduced to intersecting cones. Estimation of the aircraft's position based on a nonlinear measurement model and an underlying linear system model is achieved using a linear regression Kalman filter [1, 2]. A decomposed computation of the filter step is introduced, allowing a more efficient calculation.
\end{abstract}

Keywords: Tracking, Multilateration, Nonlinear Filtering, Estimation, Aerial surveillance.

\section{Introduction}

Multilateration techniques are chosen as a means to locate aircraft by determining distances to several receivers. If the sending time is known, the distance between transmitter and receiver can be calculated by measuring the time of arrival (TOA) and subtracting the emission time. However, the sending time is not known. The problem formulation can vary in several ways, depending of the type of measurements. Knowing the signal allows to measure receiving times at the sensors, whereas ignorance of the signal takes up correlating the signals received in order to detect similar signals, resulting in time difference of arrival (TDOA) measurements. Further distinctions can be made by defining whether the aircraft to be located is cooperative or uncooperative. In the former case, the source supports the location system by emitting signals, e.g., answering a request or autonomously emitting a signal. Uncooperative objects can be located by measuring signals reflected from their surface. The location procedure can be classified as active or passive. Active denotes that the location system interrogates the aircraft transponder, whereas passive systems listen to transmissions solicited by other equipment. Solutions can be further distinguished as being deterministic or stochastic.

In this paper, a method is presented for passively locating cooperative objects that autonomously emit signals. Furthermore, it is assumed that signals are known, allowing to measure receiving times at the sensors, and all receivers are synchronized. Common approaches use TDOAs given by subtracting the receiving times of one receiver from another, which cancels the common emission time. Each TDOA value refers to a hyperboloid in space on which the transmitter (i.e., the aircraft) must lie. Intersecting three of those yields (in the absence of any error) the aircraft's position. A new approach is to directly operate on the receiving times, as they can be interpreted geometrically as cones where the range is not perfectly known. Therefore, an additional parameter corresponding to the unknown emission time of the signal is supplied, limiting the range of the cone. Position and emission time can be retrieved by intersecting the cones. The task of intersecting hyperboloids is not as good-natured as intersecting cones and therefore, this paper concentrates on using receiving time measurements. The Gaussian estimator $[1,2]$ is used to simultaneously estimate the aircraft position and the emission time.

\subsection{State of the art}

In literature, the multilateration problem is widely addressed. Mainly, the publications are concerned with TDOA measurements. In the late 1980s, Smith and Abel proposed a closed-form solution based on a twostep least-squares procedure in $[3,4]$, which they called 
the spherical interpolation method. There, the TDOA equation is converted into equations that depend on the object's range to the origin and the position of the object. In a first step, Smith and Abel solve a linear least-squares problem assuming the position to be known, resulting in an estimation of the range. The estimated range is inserted into the equation which results in a linear least-squares problem depending on the position.

In [5], Chan and Ho propose a similar procedure which additionally takes the dependence of the range and the position of the object into account. Similar to Smith and Abel, a two-step procedure is used, where the first step corresponds to the solution that is produced by the spherical interpolation method by solving a weighted least-squares problem. The second step corrects the estimated position by inserting the dependency between range and position into a second weighted least-squares problem. The inverse of the measurement covariance matrix is used as weighting matrix.

Stoica et. al. summarize the different least-squares methods in [6] into unconstrained and constrained least-squares methods. They also introduce a new approximate least-squares method based on the use of a Taylor series expansion.

In 2006, Savage et. al. [7] proposed a stochastic solution to the multilateration problem using TDOAs in the measurement equation. They estimated the system state comprising the aircraft position, using the Unscented Kalman Filter. The benefit over the closedform solutions was the possibility of tracking a target over time as an effect of the recursive structure of the given filter.

\subsection{Paper Outline}

In Sec. 2, the abstract problem formulation is given. The measurement and system models for the considered problem are the matter of Sec. 3. The estimator equations are derived in Sec. 4 using the models derived in Sec. 3. Next, Sec. 5 compares the proposed solution to the state of the art. Finally, Sec. 6 concludes the paper.

\section{Problem Formulation}

Given receiving time measurements

$$
t_{k}(i)=\frac{\left\|\underline{s}_{i}-\underline{x}_{k}\right\|}{c}+t_{k}^{0},
$$

which result from adding the emission time $t_{k}^{0}$ and the signal runtime $\left(\left\|\underline{s}_{i}-\underline{x}_{k}\right\|\right) / c$, where $\underline{s}_{i}$ is the position of the $i$-th sensor and $\underline{x}_{k}$ denotes the target position at time $k$, with $c$ being the signal propagation speed. The current position $\underline{x}_{k}$ and the emission time $t_{k}^{0}$ are considered to be unknown and have to be determined. Fig. 1 illustrates the origin of receiving time measurements.

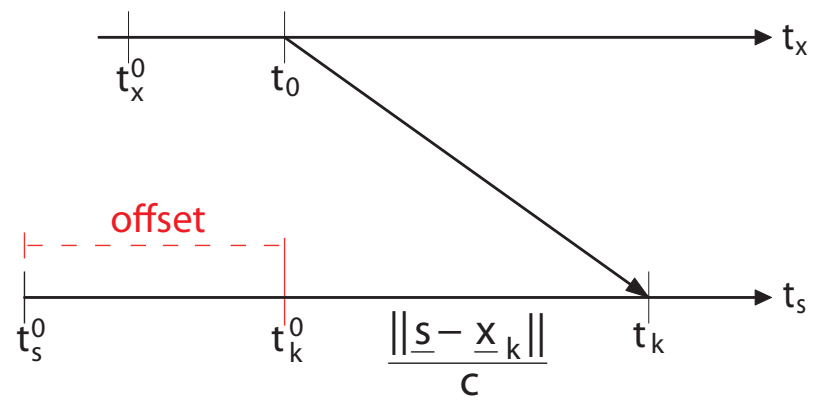

Figure 1: Illustration of a receiving time measurement. $t_{x}$ denotes local time of aircraft which emits its signal at time $t_{0}$. It takes $\left(\left\|\underline{s}-\underline{\boldsymbol{x}}_{k}\right\|\right) / c$ seconds for the signal to reach the sensor at $\underline{\boldsymbol{t}}_{k}$ relative to the local sensor time $t_{s}$. The dotted red line denotes the assumed time offset.

The emission time $t_{k}^{0}$ represents an offset in the local sensor time, which sums up with the signal runtime to form the receiving time $t_{k}$.

When the signal propagation speed $c$ is known, the receiving time measurement (1) can be converted into pseudo-range measurements

$$
y_{k}(i)=\left\|\underline{s}_{i}-\underline{x}_{k}\right\|+\underbrace{c \cdot t_{k}^{0}}_{r_{k}},
$$

where the term $r_{k}$ can be interpreted as a range offset. Given pseudo-range measurements (2), the position $\underline{\boldsymbol{x}}_{k}$ and the range offset $c \cdot \underline{\boldsymbol{t}}_{k}^{0}$ can be estimated.

Here, a stochastic estimator is used, requiring a measurement model adapted to the given measurements, and an adequate system model, which can be derived from the physical behavior of an aircraft.

\section{Modeling}

The measurements in (2) are assumed to be corrupted by additive and multiplicative noise. Besides the range measurements, the sensor positions are also considered to be uncertain. They are afflicted with additive noise, resulting in the following measurement equation,

$$
\begin{aligned}
& \left.\underline{\boldsymbol{y}}_{k}=\underline{h}_{\underline{\boldsymbol{\xi}}}, \underline{\boldsymbol{v}}_{k}^{m}\right)+\underline{\boldsymbol{v}}_{k}^{a}= \\
& {\left[\begin{array}{c}
\left\|\left(\underline{\hat{S}}_{1}+\underline{\boldsymbol{\rho}}_{k}\right)-\underline{\boldsymbol{x}}_{k}\right\| \\
\vdots \\
\left\|\left(\underline{\hat{S}}_{n}+\underline{\boldsymbol{\rho}}_{k}\right)-\underline{\boldsymbol{x}}_{k}\right\|
\end{array}\right] \cdot \underline{\boldsymbol{v}}_{k}^{m}+\underline{\boldsymbol{r}}_{k}+\underline{\boldsymbol{v}}_{k}^{a},}
\end{aligned}
$$

with $\underline{\boldsymbol{\xi}}_{k}=\left[\underline{\boldsymbol{x}}_{k}^{\mathrm{T}}, \boldsymbol{r}_{k}\right]^{\mathrm{T}}$ being the system state at time $k$ comprising the current position $\underline{\boldsymbol{x}}_{k}$ and the range offset $\boldsymbol{r}_{k}$. The random vector $\underline{\boldsymbol{v}}_{k}^{m}$ denotes a multiplicative noise process considered to be non-zero mean, white, and Gaussian distributed

$$
\underline{\boldsymbol{v}}_{k}^{m} \sim \mathcal{N}\left(\underline{1}, \mathbf{C}_{k}^{v^{m}}\right) .
$$


The $\underline{\hat{S}}_{i}$ denote the true sensor positions, which are corrupted by a measurement noise process $\underline{\rho}_{k}$ which is assumed to be zero-mean, white, and Gaussian distributed

$$
\underline{\rho}_{k} \sim \mathcal{N}\left(\underline{0}, \mathbf{C}_{k}^{\rho}\right) .
$$

The additive noise process $\underline{\boldsymbol{v}}_{k}^{a}$ denotes measurement disturbances characterized by a zero-mean, white, and Gaussian noise process

$$
\underline{\boldsymbol{v}}_{k}^{a} \sim \mathcal{N}\left(\underline{0}, \mathbf{C}_{k}^{v^{a}}\right) .
$$

The considered noise sources are thoroughly discussed in the following subsection.

\subsection{Measurement Uncertainties}

Equation (3) shows three assumed noise sources $\underline{\boldsymbol{v}}_{k}^{m}$, $\underline{\boldsymbol{v}}_{k}^{a}$, and $\underline{\boldsymbol{\rho}}_{k}$. They are due to different kinds of distortions introduced by the signal propagation as well as the sensor's properties.

\subsubsection{Sensor Uncertainty}

The sensor positions are uncertain, resulting in an error variance given by

$$
\sigma_{\rho}^{2}=\frac{1}{n^{2}} \cdot \sigma_{\rho_{0}}^{2},
$$

where $n$ denotes the number of measurements taken and $\rho_{0}$ describes the positional uncertainty of one measurement. The noise is assumed to be uncorrelated, with

$$
\mathbf{C}_{k}^{\rho}=\operatorname{diag}\left\{\sigma_{\rho}^{2}, \sigma_{\rho}^{2}, \sigma_{\rho}^{2}\right\}
$$

being the noise covariance matrix. Sensor position measurements can be performed using GNSS techniques.

Moreover, the measurements can suffer from imprecise clocks used in the sensors. Inaccuracies in synchronization of the sensors due to offset and drift are inevitable, and must therefore be considered. As the noise affects the clock devices, the measured times can be considered uncertain, which is directly influencing the ranges, resulting in an error variance $\sigma_{t}^{2}$.

\subsubsection{Signal Uncertainties}

One possible cause of uncertainty is the signal propagation, which can be influenced by reflection, diffraction, or scattering [8]. Reflection and diffraction occur if obstacles lie in the line-of-sight between the emitter and the receiver, while scattering occurs when small obstacles, in comparison with the wavelength, lie in the way of the signal. Those distortions result in errors on the measured ranges, caused by the fact that the signal propagation is no longer straight, and the range cannot be assumed to be Euclidean anymore. The resulting ranges could be longer or shorter than the true range really is. Concluding, the resulting uncertainties can be subsumed in one error component and characterized by the common error variance $\sigma_{s}^{2}$.
Both signal and sensor uncertainties are assumed to impinge directly and additively upon the measured ranges, which allows to sum up the error variances according to

$$
\mathbf{C}_{k}^{v^{a}}=\operatorname{diag}\left\{\left(\sigma_{s}^{2}+\sigma_{t}^{2}\right), \ldots,\left(\sigma_{s}^{2}+\sigma_{t}^{2}\right)\right\},
$$

forming the covariance matrix of the measurement noise $\underline{\boldsymbol{v}}_{k}^{a}$.

\subsubsection{Model uncertainty}

Due to the idealistic assumption of the signal propagation speed $c_{0}$ to be the speed of light in vacuum, a range dependent error is introduced. This range-dependent error is modeled as the multiplicative noise term $\underline{\boldsymbol{v}}_{k}^{m}$. This error can be motivated by the deviation of the true signal propagation speed and the assumed signal propagation speed. The multiplicative noise is modeled as a non-zero-mean, white Gaussian noise process

$$
\underline{\boldsymbol{v}}_{k}^{m} \sim \mathcal{N}\left(\underline{1}, \mathbf{C}_{k}^{v^{m}}\right) .
$$

The random vector $\underline{\boldsymbol{v}}_{k}^{m}$ can be decomposed into its mean and a zero-mean random vector, according to

$$
\underline{\boldsymbol{v}}_{k}^{m}=\underline{\mu}_{k}^{v^{m}}+\underline{\boldsymbol{v}}_{k}^{m_{0}} \text { with } \underline{\boldsymbol{v}}_{k}^{m_{0}} \sim \mathcal{N}\left(\underline{0}, \mathbf{C}_{k}^{v^{m}}\right) .
$$

\subsection{System Model}

The system model for the position part of the state vector $\underline{\boldsymbol{x}}_{k}$ can directly be derived from the physical behavior of the object, e.g., the aircraft, in case of aerial surveillance. Depending on the system model chosen, the noise could be correlated, resulting in a full covariance matrix $\mathbf{C}_{k}^{w}$.

Adequate aircraft motion models can be chosen depending on the expected motion to be performed. In a straight motion flight scenario, a constant velocity model [9] would be sufficient, whereas in the case of curvy flight maneuvers, a coordinated turn model $[10,11]$ is preferably used. In a realistic scenario, an aircraft never exclusively flies according to one simple model, which calls for a combination of different models, namely the interacting multiple model (IMM) [12].

Within the constant position model, no assumption is made about systematic behavior of the range offset $\boldsymbol{r}_{k}$,

$$
\boldsymbol{r}_{k+1}=\boldsymbol{r}_{k}+\boldsymbol{w}^{r},
$$

where $\boldsymbol{w}^{r}$ is assigned as white Gaussian noise with zero mean.

The constant velocity model is a linear motion model, where the system state contains the object's position and velocity

$$
\underline{\boldsymbol{x}}_{k}=\left[\begin{array}{ll}
\left(\underline{\boldsymbol{x}}_{k}^{p}\right)^{\mathrm{T}} & \left(\underline{\boldsymbol{x}}_{k}^{v}\right)^{\mathrm{T}}
\end{array}\right]^{\mathrm{T}},
$$


and the system matrix is given by

$$
\mathbf{A}_{x}=\left[\begin{array}{cc}
\mathbf{I} & T \cdot \mathbf{I} \\
\mathbf{0} & \mathbf{I}
\end{array}\right],
$$

where $\mathbf{I}$ denotes the identity matrix and $T$ is the discretization time step, $\mathbf{0}$ denotes the zero matrix. Using the system matrix, the system equation is given by

$$
\underline{\boldsymbol{x}}_{k+1}=\mathbf{A}_{x} \cdot \underline{\boldsymbol{x}}_{k}+\underline{\boldsymbol{w}}_{k}^{x},
$$

with $\underline{\boldsymbol{w}}_{k}^{x}$ being a zero-mean, Gaussian noise process with correlated covariance matrix

$$
\mathbf{C}_{x}^{w}=\left[\begin{array}{cc}
\frac{T^{3}}{3} \cdot \mathbf{Q} & \frac{T^{2}}{2} \cdot \mathbf{Q} \\
\frac{T^{2}}{2} \cdot \mathbf{Q} & T \cdot \mathbf{Q}
\end{array}\right] .
$$

The submatrix $\mathbf{Q}$ contains the positional uncertainty for each dimension on its diagonal [9]

$$
\mathbf{Q}=\operatorname{diag}\left\{\sigma_{x}^{2}, \sigma_{y}^{2}, \sigma_{z}^{2}\right\}
$$

For the whole system state, the system matrix is augmented with the constant position model for the range offset, resulting in the system matrix

$$
\mathbf{A}=\left[\begin{array}{ll}
\mathbf{A}_{x} & \underline{0} \\
\underline{0}^{\mathrm{T}} & 1
\end{array}\right],
$$

where the noise affecting the range offset is assumed to be uncorrelated with the noise on the position and velocity, with

$$
\mathbf{C}_{k}^{w}=\left[\begin{array}{ll}
\mathbf{C}_{x}^{w} & \underline{0} \\
\underline{0}^{\mathrm{T}} & \sigma_{r}^{2}
\end{array}\right]
$$

being the common covariance matrix. The system model presented is used within the experiments following in the simulations section.

\section{Estimator Design}

In this paper, a linear regression Kalman Filter, the Gaussian Estimator [1, 2], is applied. It belongs to a class of sample-based estimators, such as the Particle Filter [13] and the Unscented Kalman Filter [14, 15]. Those estimators use samples to approximate the prior density. While the Particle Filter uses random sampling, the Unscented Kalman Filter and the Gaussian Estimator use deterministic sampling methods based on the first two moments of the prior density.

In the next two sections, prediction step and filter step are derived for the given problem.

\subsection{Prediction Step}

Given the linear motion model (4), the standard Kalman prediction step can be used,

$$
\underline{\xi}_{k+1}^{p}=\mathbf{A} \cdot \underline{\xi}_{k}^{e},
$$

where $\boldsymbol{\xi}$ is the system state as introduced in Sec. 3, superscript $p$ denotes statistics of the predicted density and $e$ is the estimated density. The covariance matrix of the predicted density can be computed using

$$
\mathbf{C}_{k+1}^{p}=\mathbf{A} \cdot \mathbf{C}_{k}^{e} \cdot \mathbf{A}^{\mathrm{T}}+\mathbf{C}_{k}^{w}
$$

where the uncertainty of the predicted density is increased by adding the covariance matrix of the system noise $\mathbf{C}_{k}^{w}$.

When using a nonlinear system model, the prior density has to be sampled and the prediction step is performed using the samples, according to the paradigm of the Gaussian Estimator [1].

\subsection{Filter Step}

Assuming the nonlinear measurement model (3), the predicted density needs to be approximated. Under the Gaussian assumption the first two moments of the densities represent sufficient statistics. The samples are computed deterministically using the mean and the covariance matrix of the given predicted density. The filter step will be performed in terms of the mean

$$
\underline{\xi}_{k}^{e}=\underline{\xi}_{k}^{p}+\mathbf{C}_{k}^{\xi, y} \cdot\left(\mathbf{C}_{k}^{y}\right)^{-1} \cdot\left(\underline{y}-\underline{\mu}_{y}\right) .
$$

and covariance matrix

$$
\begin{aligned}
& \mathbf{C}_{k}^{e}=\mathbf{C}_{k}^{p}- \\
& \quad \mathbf{C}_{k}^{\xi, y} \cdot\left(\mathbf{C}_{k}^{y}\right)^{-1} \cdot \mathbf{C}_{k}^{y} \cdot\left(\mathbf{C}_{k}^{\xi, y} \cdot\left(\mathbf{C}_{k}^{y}\right)^{-1}\right)^{\mathrm{T}},
\end{aligned}
$$

of the posterior density. To solve equations (5), and (6) the measurement covariance matrix $\mathbf{C}_{k}^{y}$ and the cross covariance matrix $\mathbf{C}_{k}^{\xi, y}$ are to be known.

\subsubsection{State Decomposition}

The system state is decomposed according to the fact that the measurements only depend partly on the system state. Using the constant velocity model from the example the two state parts result in

$$
\begin{aligned}
& \underline{\boldsymbol{x}}_{k}^{a}=\left[\begin{array}{ll}
\left(\underline{\boldsymbol{x}}_{k}^{p}\right)^{\mathrm{T}} & \boldsymbol{r}_{k}
\end{array}\right]^{\mathrm{T}}, \\
& \underline{\boldsymbol{x}}_{k}^{b}=\underline{\boldsymbol{x}}_{k}^{v}
\end{aligned}
$$

where the state density can be decomposed according to Bayes' law, resulting in

$$
f\left(\underline{\boldsymbol{x}}_{k}^{b} \mid \underline{\boldsymbol{x}}_{k}^{a}\right) \cdot f\left(\underline{\boldsymbol{x}}_{k}^{a}\right) .
$$

The prior density $f\left(\underline{\boldsymbol{\xi}}_{k}\right)$ is assumed to be Gaussian with covariance matrix

$$
\mathbf{C}_{k}^{\xi}=\left[\begin{array}{cc}
\mathbf{C}_{k}^{a} & \mathbf{C}_{k}^{a, b} \\
\mathbf{C}_{k}^{b, a} & \mathbf{C}_{k}^{b}
\end{array}\right]
$$

The Gaussian assumption leads to a Gaussian distributed state part $\underline{\boldsymbol{x}}_{k}^{a}$, given by

$$
\mathcal{N}\left(\underline{\boldsymbol{x}}_{k}^{a}-\underline{\mu}_{k}^{a}, \mathbf{C}_{k}^{a}\right),
$$


corresponding to $f\left(\underline{\boldsymbol{x}}_{k}^{a}\right)$. Out of $f\left(\underline{\boldsymbol{x}}_{k}^{b} \mid \underline{\boldsymbol{x}}_{k}^{a}\right)$, the density of $\underline{\boldsymbol{x}}_{k}^{b}$ can be given as Gaussian density [2] with

$$
\begin{aligned}
\mathcal{N}\left(\underline{\boldsymbol{x}}_{k}^{b}-\left[\underline{\mu}_{k}^{b}+\mathbf{C}_{k}^{b, a} \cdot\left(\mathbf{C}_{k}^{a}\right)^{-1} \cdot\left(\underline{\boldsymbol{x}}_{k}^{a}-\underline{\mu}_{k}^{b}\right)\right],\right. \\
\left.\mathbf{C}_{k}^{b}-\mathbf{C}_{k}^{b, a} \cdot\left(\mathbf{C}_{k}^{a}\right)^{-1} \cdot \mathbf{C}_{k}^{a, b}\right) .
\end{aligned}
$$

\subsubsection{Measurement Covariance Matrix}

The predicted state density is approximated in accordance with the Gaussian Estimator. Caused by the fact, that the measurement model only depends on the part $\underline{\boldsymbol{x}}_{k}^{a}$ of the system state, merely the density $f\left(\underline{\boldsymbol{x}}_{k}^{a}\right)$ needs to be sampled. The approximation is carried out using a Dirac Mixture density [1]

$$
f\left(\underline{\boldsymbol{x}}^{a}\right) \approx \frac{1}{L} \cdot \sum_{i=1}^{L} \delta\left(\underline{\boldsymbol{x}}^{a}-\underline{\mu}_{i}^{a}\right),
$$

with the positions $\mu_{i}^{a}$ of the Dirac components being the samples of the approximated density. By propagating the samples through the measurement model (3), the samples

$$
\underline{\mu}_{i}^{y}=\underline{h}\left(\underline{\mu}_{i}^{a}\right)
$$

corresponding to the measurement density $f\left(\underline{\boldsymbol{y}}_{k}\right)$ are computed. According to [1] the first and second moment of the approximated density are preserved and can be retrieved using the sample mean and the sample covariance, resulting in the mean

$$
\underline{\mu}_{k}^{y}=\frac{1}{L} \cdot \sum_{i=1}^{L} \underline{\mu}_{i}^{y},
$$

where $L$ denotes the total number of components used to approximate the density, and the measurement covariance

$$
\mathbf{C}_{k}^{y}=\frac{1}{d} \cdot \sum_{i=1}^{L}\left(\underline{\mu}_{i}^{y}-\underline{\mu}_{k}^{y}\right) \cdot\left(\underline{\mu}_{i}^{y}-\underline{\mu}_{k}^{y}\right)^{\mathrm{T}}+\mathbf{C}_{k}^{v},
$$

where the samples are weighted with respect to the number of components used to approximate one dimension of the density.

\subsubsection{Cross Covariance Matrix}

Finally the cross covariance has to be computed using

$$
\begin{aligned}
\mathbf{C}_{k}^{\xi, y}=\iint\left(\underline{\boldsymbol{\xi}}_{k}-\underline{\mu}_{k}^{\xi}\right) \cdot\left(\underline{\boldsymbol{y}}_{k}-\underline{\mu}_{k}^{y}\right)^{\mathrm{T}} . & \\
& f\left(\underline{\boldsymbol{y}}_{k} \mid \underline{\boldsymbol{\xi}}_{k}\right) f\left(\underline{\boldsymbol{\xi}}_{k}\right) \mathrm{d} \underline{\boldsymbol{\xi}}_{k} \mathrm{~d} \underline{\boldsymbol{y}}_{k},
\end{aligned}
$$

according to the decomposition into observable and unobservable parts of the system state [2]. Under the decomposition the cross covariance matrix is given by

$$
\mathbf{C}_{k}^{\xi, y}=\left[\begin{array}{l}
\mathbf{C}_{k}^{a, y} \\
\mathbf{C}_{k}^{b, y}
\end{array}\right]
$$

The computation of $\mathbf{C}_{k}^{a, y}$ is performed using the samples from (7) and the prior mean, as well as the samples from the measurement density (8) and the resulting mean. By inserting the samples and the means into the sample covariance equation the cross covariance matrix depending on the state $\underline{\boldsymbol{x}}_{k}^{a}$ resolves to

$$
\mathbf{C}_{k}^{a, y}=\frac{1}{d} \cdot \sum_{i=1}^{L}\left(\underline{\mu}_{i}^{a}-\underline{\mu}_{x}^{a}\right) \cdot\left(\underline{\mu}_{i}^{y}-\underline{\mu}_{k}^{y}\right)^{\mathrm{T}} .
$$

The cross covariance matrix $\mathbf{C}_{k}^{b, y}$ corresponding to $\underline{\boldsymbol{x}}_{k}^{b}$ is evaluated analytically, using

$$
\begin{array}{r}
\mathbf{C}_{k}^{b, y}= \\
\iint \underline{\boldsymbol{x}}_{k}^{b} \cdot \underline{h}\left(\underline{\boldsymbol{x}}_{k}^{a}\right)^{\mathrm{T}} \cdot f\left(\underline{\boldsymbol{x}}_{k}^{b} \mid \underline{\boldsymbol{x}}_{k}^{a}\right) \cdot f\left(\underline{\boldsymbol{x}}_{k}^{a}\right) \mathrm{d} \underline{\boldsymbol{x}}_{k}^{a} \mathrm{~d} \underline{\boldsymbol{x}}_{k}^{b}- \\
\underline{\mu}_{k}^{b} \cdot\left(\underline{\mu}_{k}^{y}\right)^{\mathrm{T}} .
\end{array}
$$

By marginalizing $\underline{\boldsymbol{x}}_{k}^{b}$, rearranging the integrals, and analytically evaluating the integrals, the cross covariance matrix is given by

$$
\begin{aligned}
& \mathbf{C}_{k}^{b, y}= \underline{\mu}_{k}^{b} \cdot\left(\underline{\mu}_{k}^{y}\right)^{\mathrm{T}}+ \\
& \mathbf{C}_{k}^{b, a} \cdot\left(\mathbf{C}_{k}^{a}\right)^{-1} \cdot\left[\mathbf{C}_{k}^{a, y}+\underline{\mu}_{k}^{a} \cdot\left(\underline{\mu}_{k}^{y}\right)^{T}\right]- \\
& \\
& \quad\left[\mathbf{C}_{k}^{b, a} \cdot\left(\mathbf{C}_{k}^{a}\right)^{-1} \cdot \underline{\mu}_{k}^{a} \cdot\left(\underline{\mu}_{k}^{y}\right)^{\mathrm{T}}\right]-\underline{\mu}_{k}^{b} \cdot\left(\underline{\mu}_{k}^{y}\right)^{\mathrm{T}},
\end{aligned}
$$

which simplifies to

$$
\mathbf{C}_{k}^{b, y}=\mathbf{C}_{k}^{b, a} \cdot\left(\mathbf{C}_{k}^{a}\right)^{-1} \cdot \mathbf{C}_{k}^{a, y}
$$

by deliberately adding the components.

The full cross covariance can be computed by inserting the results into (9), which simplifies the computation of the filter step. By using the proposed decomposition approach, only the partial density $f\left(\underline{\boldsymbol{x}}_{k}^{a}\right)$ needs to be sampled, conserving computation power.

\section{Simulation Results}

The proposed estimator for the multilateration problem is compared to state of the art methods using three simulations. All of them are performed using the same trajectory over 250 time steps. The trajectory is shown in Fig. 2 as horizontal projection. Due to the fact that all sensors are nearly lying on a plane, the true height cannot be accurately reconstructed. In the simulations, only the horizontal positioning error is considered. The sensors are placed around the sample trajectory, located at the positions given in table 1 .

The first simulation concentrates on different standard deviations for the measurement noise and shows how state of the art methods as well as the proposed estimator perform under these conditions. In the second simulation, the number of sensors is varied in order to show the estimator's performance. In the third simulation the robustness using subspace measurements is 


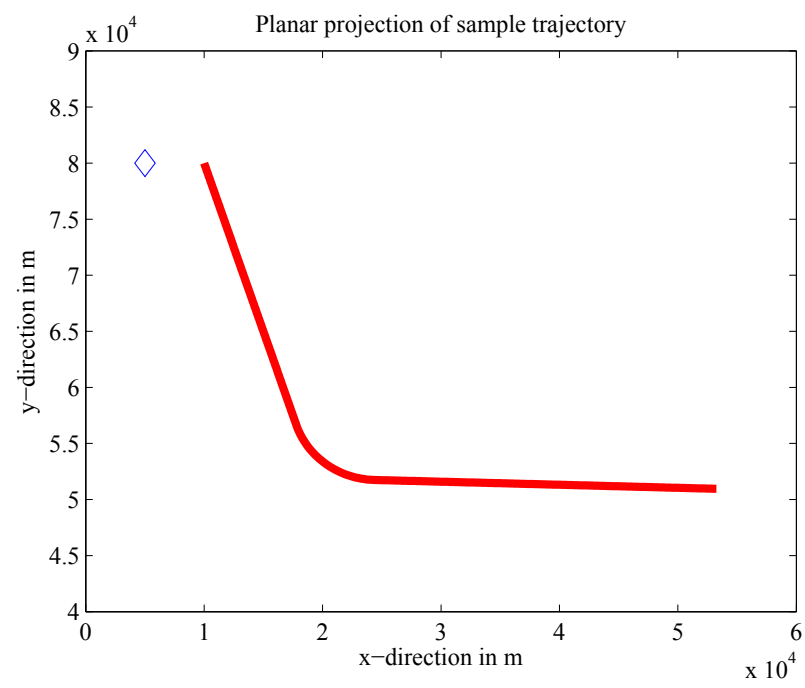

Figure 2: A planar projection of the aircraft's trajectory is shown in red. The blue diamond denotes the sensor that is located nearest to the trajectory.

Table 1: Sensor positions.

\begin{tabular}{l|rrr} 
Sensor & $\mathrm{x} / \mathrm{km}$ & $\mathrm{y} / \mathrm{km}$ & $\mathrm{z} / \mathrm{km}$ \\
\hline $\mathrm{A}$ & 10 & 10 & 0.150 \\
$\mathrm{~B}$ & 30 & 120 & 0.360 \\
$\mathrm{C}$ & 100 & 35 & 0.220 \\
$\mathrm{D}$ & 175 & 110 & 0.060 \\
$\mathrm{E}$ & 200 & 75 & 0.140 \\
$\mathrm{~F}$ & 5 & 80 & 0.420 \\
$\mathrm{G}$ & 170 & 10 & 0.270
\end{tabular}

shown. Due to the least-squares approaches' disability to use subspace measurements, only the proposed estimator and the Unscented Kalman Filter from [7] are used.

\subsection{Simulation 1: Noise levels}

The measurements of the sample trajectory are corrupted by noise with different standard deviations. The values used for the standard deviation are $0.3 \mathrm{~m}, 3 \mathrm{~m}$, $7.5 \mathrm{~m}$, and $15 \mathrm{~m}$. Among different noise levels, the proposed Gaussian Estimator using receiving time measurements is compared to the state of the art represented by the Unscented Kalman Filter using TDOA measurements [7], the Spherical Interpolation method [3], and the hyperbolic location algorithm by Chan and Ho [5].

The RMSE and the standard deviation of the error is evaluated over 1000 Monte Carlo simulations. The results concerning the RMSE are given in Table 2, and the standard deviations of the resulting errors are shown in Table 3. Fig. 3 shows the RMSE values given the different noise standard deviations.

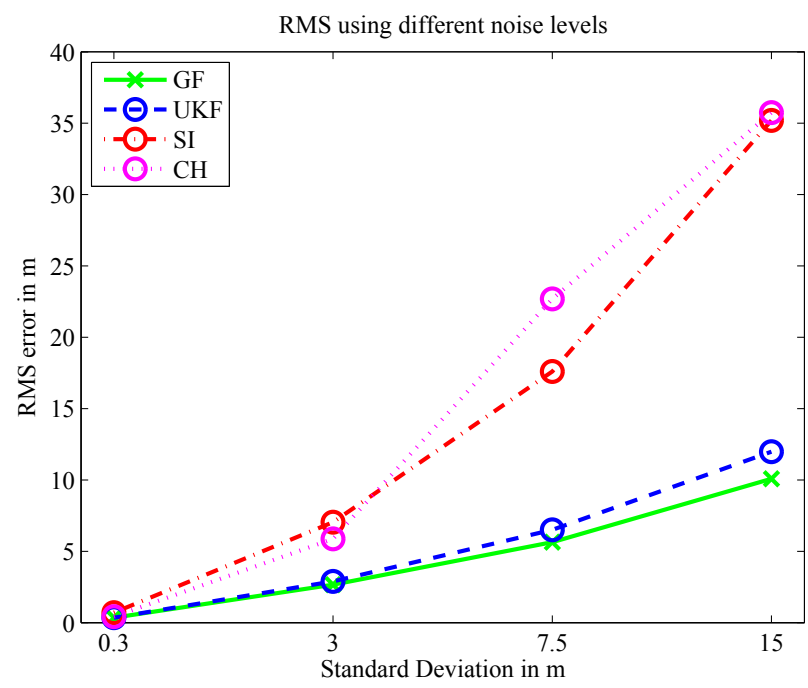

Figure 3: The mean RMSEs under changing measurement noise standard deviations is shown. The GF (green, dotted) shows the best results, while the UKF (blue, dashed) shows almost as good results as the GF. The SI (red, dot-dashed) and the CH (magenta, dotted) show minor noise immunity.

Table 2: RMS errors from noisy measurements.

\begin{tabular}{l|cccc} 
Noise & $0.3 \mathrm{~m}$ & $3 \mathrm{~m}$ & $7.5 \mathrm{~m}$ & $15 \mathrm{~m}$ \\
\hline GF & 0.3491 & 2.6538 & 5.6383 & 10.0773 \\
UKF & 0.3488 & 2.8907 & 6.5087 & 11.9764 \\
SI & 0.7040 & 7.0403 & 17.6004 & 35.1999 \\
CH & 0.4247 & 5.8743 & 22.6892 & 35.7477
\end{tabular}

As a result, the Gaussian Estimator using receiving time measurements outperforms the examined state of the art methods with increasing noise levels.

\subsection{Simulation 2: Different Sensor Count}

In this simulation, a constant standard deviation of 7.5 $\mathrm{m}$ for the noise corrupting the measurements is used. Here, only the number of available sensors is changed. For each number of sensors, 1000 Monte Carlo runs are performed, where the specific sensors are chosen randomly. Five different numbers of sensors are used, namely $3,4,5,6$, and 7 .

Here, the proposed estimator is compared to all of the state of the art methods. In Fig. 4, the resulting

Table 3: Standard deviation from noisy measurements.

\begin{tabular}{l|cccc} 
Noise & $0.3 \mathrm{~m}$ & $3 \mathrm{~m}$ & $7.5 \mathrm{~m}$ & $15 \mathrm{~m}$ \\
\hline GF & 0.2638 & 1.7754 & 3.6941 & 6.2075 \\
UKF & 0.2561 & 2.0726 & 4.6832 & 8.4309 \\
SI & 0.4731 & 4.7306 & 11.8250 & 23.6454 \\
CH & 0.3213 & 11.5373 & 43.6226 & 48.9083
\end{tabular}


horizontal RMSE is shown given the different numbers of sensors. Tables 4 and 5 show the RMSE values and the resulting standard deviation of the RMSEs for the given estimators.

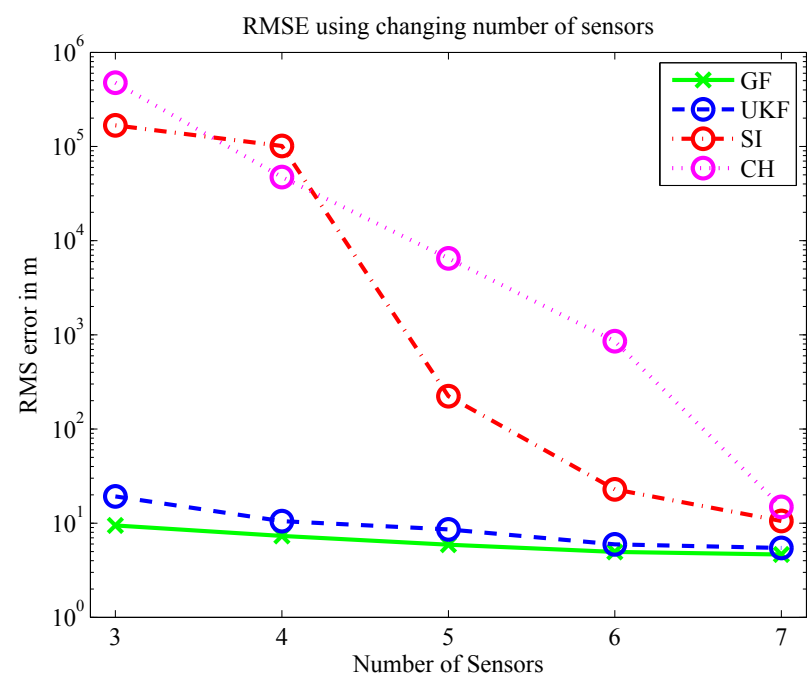

Figure 4: The RMSE with respect to the number of sensors used is shown. Logarithmic scale is used for the RMSE caused by the poor results of the least-squares approaches using too few sensors. The proposed Gaussian Estimator (green, solid) achieves best results, while the UKF (blue, dashed) is working almost as good as the GF. The SI (red, dot-dashed) shows poor results, as well as the $\mathrm{CH}$ (magenta, dotted).

Table 4: RMS errors from different numbers of sensors.

\begin{tabular}{l|ccccc} 
& $\# 3$ & $\# 4$ & $\# 5$ & $\# 6$ & $\# 7$ \\
\hline & & & & & \\
$\mathrm{GF}$ & 9.4469 & 7.3130 & 5.9102 & 4.9533 & 4.6424 \\
$\mathrm{UKF}$ & 19.208 & 10.494 & 8.5928 & 5.9561 & 5.4407 \\
$\mathrm{SI}$ & $1.68 e^{5}$ & $1.02 e^{5}$ & 223.45 & 22.831 & 10.552 \\
$\mathrm{CH}$ & $4.77 e^{5}$ & $4.74 e^{4}$ & $6.48 e^{3}$ & 854.73 & 14.873
\end{tabular}

While the least-squares methods perform poorly using less than 5 sensors, the Gaussian Estimator as well as the Unscented Kalman Filter maintain robustness using a smaller number of sensors. Again, the proposed estimator performs better than the Unscented Kalman Filter.

\subsection{Simulation 3: Sensor Failure}

The last simulation concentrates on the robustness of the estimators under the influence of sensor failures. Here, only the stochastic estimators are used due to the fact that the least-squares approaches fail using fewer than four measurements. A standard deviation of $7.5 \mathrm{~m}$ for the measurement noise is assumed.

Two Monte Carlo runs with 1000 trials are performed, in which a sensor failure probability of $10 \%$
Table 5: Standard deviation from different sensor numbers.

\begin{tabular}{l|ccccc} 
& $\# 3$ & $\# 4$ & $\# 5$ & $\# 6$ & $\# 7$ \\
\hline & & & & & \\
GF & 6.3591 & 4.2699 & 3.3263 & 2.7136 & 2.6194 \\
$\mathrm{UKF}$ & 17.080 & 7.9058 & 6.3253 & 3.7703 & 3.2189 \\
$\mathrm{SI}$ & $8.24 e^{5}$ & $4.42 e^{5}$ & $1.41 e^{3}$ & 28.262 & 6.8524 \\
$\mathrm{CH}$ & $4.28 e^{6}$ & $1.17 e^{5}$ & $2.23 e^{4}$ & $4.12 e^{3}$ & 38.915
\end{tabular}

in the first run and a probability of $20 \%$ in the second run is assigned for each sensor. The results are shown as means of the RMSE of the estimates and the corresponding standard deviations in Fig. 5. The explicit values are given in Tables 6 and 7 .

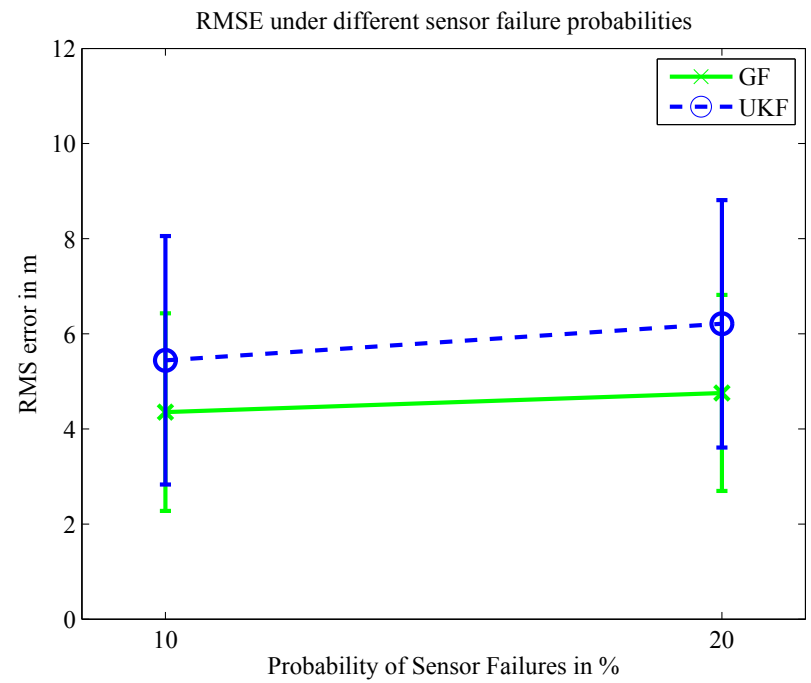

Figure 5: Error bar plots showing the mean RMSE over 1000 runs under a failing sensor scenario. The first values denote the mean RMSE and the corresponding standard deviation under a failing probability of $10 \%$, while the second values are based on a failing probability of $20 \%$. The GF (green, solid) using receiving time measurements performs slightly better than the UKF (blue, dashed) using TDOA measurements.

Table 6: RMS errors from sensor failures.

\begin{tabular}{l|cc} 
& $10 \%$ & $20 \%$ \\
\hline & & \\
GF & 4.3533 & 4.7563 \\
UKF & 5.4419 & 6.2111
\end{tabular}

The results show superior robustness of the proposed estimator in the presence of subspace measurements.

\section{Conclusions}

Most efforts to perform multilateration tracking are focused on the use of TDOA measurements. In this contribution, however, a system is introduced to directly 
Table 7: Standard deviations from sensor failures.

\begin{tabular}{l|cc} 
& $10 \%$ & $20 \%$ \\
\hline & & \\
$\mathrm{GF}$ & 2.0765 & 2.0600 \\
$\mathrm{UKF}$ & 2.6125 & 2.6013
\end{tabular}

operate on the receiving times, allowing for simultaneous estimation of aircraft position and range to the sensors. Therefore, the aircraft's emission time is estimated as an offset that is common to all sensors. Using just receiving times, the multilateration process is simplified, as cones are intersected instead of hyperboloids. The prediction takes a linear system model as a basis, while the filter step makes use of a decomposition into a directly observed part and an unobserved part. The decomposed computation of the cross-covariance matrix is shown, using an analytic and an approximate computation depending on the part of the system state. As experiments show, greater robustness towards noise and sensor failures is achieved compared to state of the art methods. Decomposition techniques are applied to increase efficiency within the filter step, while the use of the Gaussian filter [1,2] significantly improves performance.

\section{References}

[1] M. F. Huber and U. D. Hanebeck, "Gaussian Filter based on Deterministic Sampling for High Quality Nonlinear Estimation," in Proceedings of the 17th IFAC World Congress (IFAC 2008), vol. 17, no. 2, Seoul, Korea, July 2008.

[2] F. Beutler, M. F. Huber, and U. D. Hanebeck, "Gaussian Filtering using State Decomposition Methods," in Proceedings of the 12th International Conference on Information Fusion (Fusion 2009), Seattle, Washington, July 2009.

[3] J. S. Abel and J. O. Smith, "The Spherical Interpolation Method for Closed-Form Passive Source Localization using Range Difference Measurements," IEEE Transactions on Acoustics, Speech, and Signal Processing, vol. 12, pp. 471-474, April 1987.

[4] J. O. Smith and J. S. Abel, "The Spherical Interpolation Method of Source Localization," IEEE Journal of Oceanic Engineering, vol. OE-12, no. 1, pp. 246-252, January 1987.

[5] Y. T. Chan and K. C. Ho, "A Simple and Efficient Estimator for Hyperbolic Location," IEEE Transactions on Signal Processing, vol. 42, no. 8, pp. 1905-1915, August 1994.
[6] P. Stoica and J. Li, "Source Localization from Range-Difference Measurements," IEEE Signal Processing Magazine, vol. 1, pp. 63-65, November 2006.

[7] C. O. Savage, R. L. Cramer, and H. A. Schmitt, "TDOA Geolocation with the Unscented Kalman Filter," Networking, Sensing and Control, 2006. ICNSC '06. Proceedings of the 2006 IEEE International Conference on, pp. 602-606, 2006.

[8] T. S. Rappaport, Wireless Communications, Principles and Practice, Second Edtion. Prentice Hall PTR, 2002.

[9] G. Welch, A. Ilie, G. Bishop, and B. D. Allen, "Measurement Sample Time Optimization for Human Motion Tracking/Capture Systems," in Proceedings of Trends and Issues in Tracking for Virtual Environments, Workshop at the IEEE Virtual Reality 2007 Conference (Charlotte, NC USA), G. Zachmann, Ed., 2007.

[10] M. R. Morelande and N. J. Gordon, "Target Tracking Through A Coordinated Turn," Proceedings of IEEE International Conference on Acoustics, Speech and Signal Processing, vol. 4, pp. iv/21iv/24, March 2005.

[11] X. Yuan, C. Han, Z. Duan, and M. Lei, "Comparison and Choice of Models in Tracking Target with Coordinated Turn Motion," 8th International Conference on Information Fusion, vol. 2, p. 6pp, July 2005.

[12] Y. Bar-Shalom, X. R. Li, and T. Kirubarajan, Estimation with Applications to Tracking and Navigation. John Wiley \& Sons, Inc., 2001.

[13] M. S. Arulampalam, S. Maskell, N. Gordon, and T. Clapp, "A Tutorial on Particle Filters for On-line Non-linear/Non-Gaussian Bayesian Tracking," IEEE Transactions on Signal Processing, vol. 50, no. 2, pp. 174-188, 2002.

[14] S. Julier and J. Uhlmann, "A New Extension of the Kalman Filter to Nonlinear Systems," in Proceedings of SPIE AeroSense, 11th International Symposium on Aerospace/Defense Sensing, Simulation, and Controls, Orlando, FL, 1997.

[15] S. Julier, J. Uhlmann, and H. F. Durrant-Whyte, "A New Method for the Nonlinear Trannsformation of Means and Covariances in Filters and Estimators," IEEE Transactions on Automatic Control, vol. 45, no. 3, pp. 477-482, 2000. 